\title{
ELIXIR-IT HPC@CINECA: high performance computing resources for the bioinformatics community
}

\author{
Tiziana Castrignanò ${ }^{1 *}$, Silvia Gioiosa ${ }^{2,3}$, Tiziano Flati ${ }^{2,3}$, Mirko Cestari ${ }^{2}$, Ernesto Picardi ${ }^{3,4}$, Matteo Chiara ${ }^{3,5}$, \\ Maddalena Fratelli ${ }^{6}$, Stefano Amente ${ }^{7}$, Marco Cirilli, ${ }^{8}$, Marco Antonio Tangaro ${ }^{3}$, Giovanni Chillemi ${ }^{3,9}$, \\ Graziano Pesole $e^{3,4^{*}}$ and Federico Zambelli, ${ }^{3,5^{*}}$
}

From 13th Bioinformatics and Computational Biology Conference - BBCC 2018

Naples, Italy. 19-21 November 2018

\author{
* Correspondence: tiziana. \\ castrignano@unitus.ti; graziano. \\ pesole@uniba.it; federico.zambelli@ \\ unimi.it \\ 'Department of Ecological and \\ Biological Sciences (DEB), University \\ of Tuscia, Viterbo, Italy \\ ${ }^{3}$ Institute of Biomembranes, \\ Bioenergetics and Molecular \\ Biotechnologies, National Research \\ Council (IBIOM-CNR), Bari, Italy \\ Full list of author information is \\ available at the end of the article
}

\begin{abstract}
Background: The advent of Next Generation Sequencing (NGS) technologies and the concomitant reduction in sequencing costs allows unprecedented high throughput profiling of biological systems in a cost-efficient manner. Modern biological experiments are increasingly becoming both data and computationally intensive and the wealth of publicly available biological data is introducing bioinformatics into the "Big Data" era. For these reasons, the effective application of High Performance Computing (HPC) architectures is becoming progressively more recognized also by bioinformaticians.

Here we describe HPC resources provisioning pilot programs dedicated to bioinformaticians, run by the Italian Node of ELIXIR (ELIXIR-IT) in collaboration with CINECA, the main Italian supercomputing center.

Results: Starting from April 2016, CINECA and ELIXIR-IT launched the pilot Call "ELIXIR-IT HPC@CINECA", offering streamlined access to HPC resources for bioinformatics. Resources are made available either through web front-ends to dedicated workflows developed at CINECA or by providing direct access to the High Performance Computing systems through a standard command-line interface tailored for bioinformatics data analysis. This allows to offer to the biomedical research community a production scale environment, continuously updated with the latest available versions of publicly available reference datasets and bioinformatic tools. Currently, 63 research projects have gained access to the HPC@CINECA program, for a total handout of $\sim 8$ Millions of CPU/hours and, for data storage, 100 TB of permanent and $~ 300$ TB of temporary space.

(Continued on next page)
\end{abstract}

\section{$\triangle B M C$}

(c) The Author(s). 2020 Open Access This article is licensed under a Creative Commons Attribution 4.0 International License, which permits use, sharing, adaptation, distribution and reproduction in any medium or format, as long as you give appropriate credit to the original author(s) and the source, provide a link to the Creative Commons licence, and indicate if changes were made. The images or other third party material in this article are included in the article's Creative Commons licence, unless indicated otherwise in a credit line to the material. If material is not included in the article's Creative Commons licence and your intended use is not permitted by statutory regulation or exceeds the permitted use, you will need to obtain permission directly from the copyright holder. To view a copy of this licence, visit http://creativecommons.org/licenses/by/4.0/. The Creative Commons Public Domain Dedication waiver (http://creativecommons.org/publicdomain/zero/1.0/) applies to the data made available in this article, unless otherwise stated in a credit line to the data. 
(Continued from previous page)

Conclusions: Three years after the beginning of the ELIXIR-IT HPC@CINECA program, we can appreciate its impact over the Italian bioinformatics community and draw some considerations. Several Italian researchers who applied to the program have gained access to one of the top-ranking public scientific supercomputing facilities in Europe. Those investigators had the opportunity to sensibly reduce computational turnaround times in their research projects and to process massive amounts of data, pursuing research approaches that would have been otherwise difficult or impossible to undertake. Moreover, by taking advantage of the wealth of documentation and training material provided by CINECA, participants had the opportunity to improve their skills in the usage of HPC systems and be better positioned to apply to similar EU programs of greater scale, such as PRACE. To illustrate the effective usage and impact of the resources awarded by the program - in different research applications we report five successful use cases, which have already published their findings in peer-reviewed journals.

Keywords: HPC, Compute service, Bioinformatics, Software environment, NGS data analysis

\section{Background}

The amount of data generated by various scientific experimental platforms is growing exponentially with modern high-throughput technologies. In the case of life science, with the advent of new Next-Generation Sequencing (NGS) technologies [1] and their many applications (e.g, [2-5]), we witnessed to what is defined as a biological data deluge [6]. This large data flux introduces de-facto bioinformatics into the "big data" era [7], however the analysis and interpretation of these massive amounts of data entails relevant computational challenges.

The number of research groups working with NGS data has grown continuously in the last few years and, accordingly, their needs in terms of computational and storage facilities are constantly increasing. It's becoming more and more common that the hardware requirements needed to perform a bioinformatic analysis in a convenient frame of time far exceed those available in desktop computers, servers, and even local IT facilities. As a result, biologists and bioinformaticians are increasingly making use of HPC architectures made available by supercomputing centers [8].

High performance computing availability is thus becoming a key factor in making effective use of high throughput sequencing (HTS) biological data, that is extracting meaningful biological information in $\mathrm{a}^{1}$ reasonable amount of time. The typical scenario involves biological datasets that can easily include billions or even trillions of NGS reads and hundreds or thousands of samples, involving a storage occupancy size up to hundreds of TB per experiment or research project (e.g. MEGA, the European Alliance for Personalized Medicine). Furthermore, most use cases require complex analytical workflows implying the execution of multiple steps and the usage of severalopen source or proprietary- bioinformatics tools. The number of samples included in an analysis can be further increased by the re-analysis of similar experiments previously conducted by other research groups and accessible through web archives storing HTS data (e.g. The Sequence Read Archive SRA ${ }^{1}$, the Cancer Genome Atlas TCGA, ${ }^{2}$ the

\footnotetext{
${ }^{1}$ https://www.ncbi.nlm.nih.gov/sra Accessed 18 Mar 2020

2 https://tcga-data.nci.nih.gov Accessed 18 Mar 2020
} 
Genotype-Tissue Expression GTex ${ }^{3}$ among others) in order to compare and validate the results obtained.

However, it must be also noted that the majority of currently available bioinformatics tools are not yet developed nor optimized with HPC in mind, therefore their availability on generic HPC facilities is still limited. As a consequence, most life science researchers and bioinformatics software users and developers are not used to interact with public HPC facilities and make a limited use of the ones available to them. Offering an HPC service tailored for bioinformatics analyses can therefore provide relevant advantages that go beyond the possibility of analysing massive amounts of biological data in a timely manner. In fact, it introduces life science researchers to the HPC work environment, raising their awareness of the opportunities offered by HPC and thus making more likely the future development of tools, algorithms, applications and implementations optimized for HPC environments possibly stemming a positive feedback loop.

\section{Implementation}

\section{The ELIXIR-IT HPC@CINECA call: HPC resources for the bioinformatics community}

ELIXIR-IT constitutes the Italian Node of ELIXIR, an intergovernmental organisation that brings together life science computational infrastructures and resources from across Europe with the objective of creating a homogenous and seamless infrastructure for biological data. ELIXIR-IT is structured as a Joint Research Unit (JRU) named ELIXIR-IIB (Infrastruttura Italiana di Bioinformatica - Italian Infrastructure for Bioinformatics), and currently involves 23 partners among Universities and Research Institutions/Facilities of national relevance and led by the National Research Council (CNR).

On april 2016, ELIXIR-IT and CINECA, one of the founding members of the JRU, launched a pilot project named ELIXIR-IT HPC@CINECA, aimed at providing an entry-level -but still substantial- package of HPC resources ( $50 \mathrm{k}$ core hours, $1 \mathrm{~TB}$ of permanent storage extensible depending on the project needs) to research projects presented by Italian and European life science researchers. Three years after its inception, we can evaluate the impact of this initiative that, with over 60 project proposals submitted, an acceptance rate of about $90 \%$ and several publications made possible by the HPC resources assigned, can now be considered as a successful experimental service.

Project proposals are submitted to HPC@CINECA through a simple dedicated online form ${ }^{4}$, where the applicants are required to describe relevant aspects of their project including the motivations that make access to an HPC facility convenient. Subsequently, a technical and scientific evaluation committee (see below), evaluates the project to assess its technical feasibility and scientific soundness. Upon approval, the successful applicant gain access to the CINECA infrastructure either i) through a command-line interface (for those who need flexibility and customizable components) or ii) through a set of predefined web pipelines (for those who are not confident with command line and identify in such packaged solutions valid tools for their analyses). If needed, users can also request the installation of specific software or reference dataset not yet available on the CINECA HPC bioinformatic environment. All the new software is installed on the system by an expert support team and made available to all the users of the

${ }^{3}$ https://commonfund.nih.gov/GTEx Accessed 18 Mar 2020 


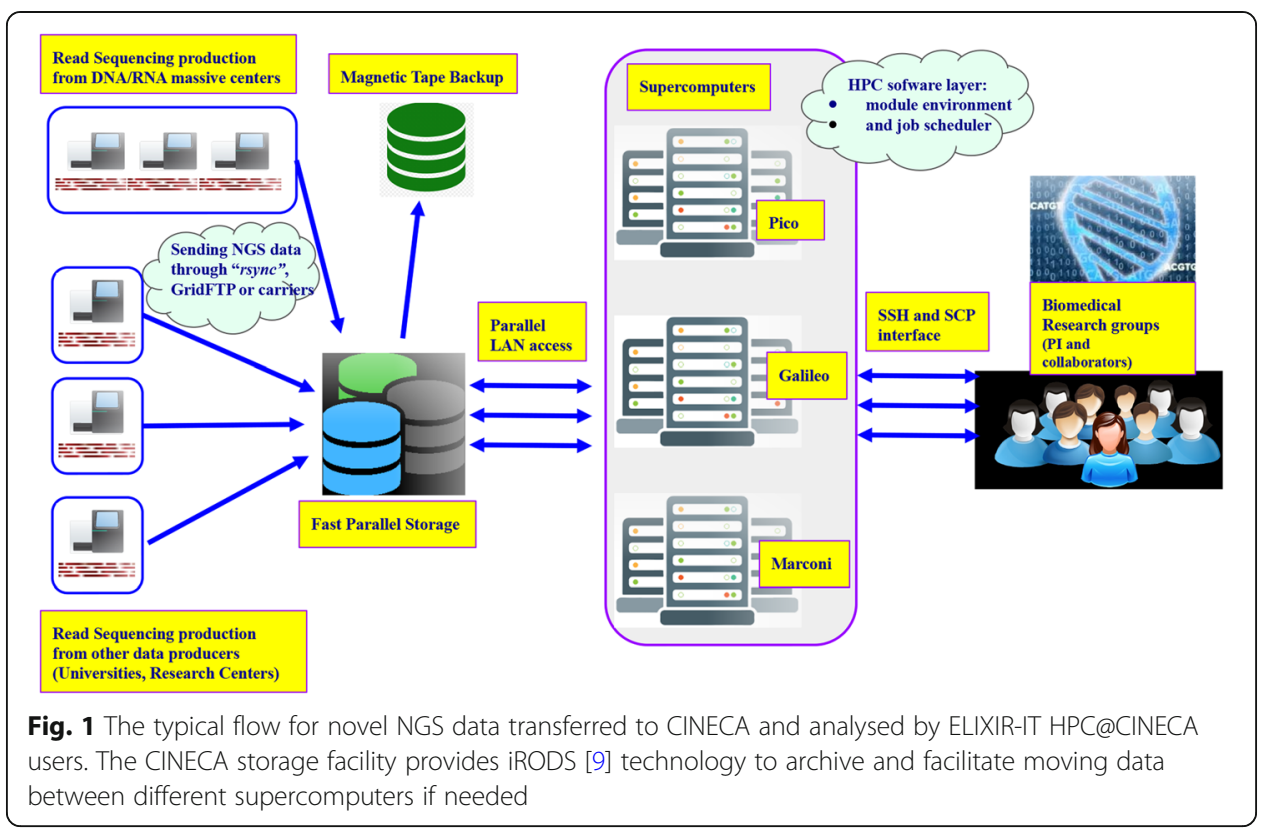

HPC platform. This mechanism helps in keeping the CINECA HPC work environment continuously up-to-date with the most widely used software tools and reference datasets (for further details refer to the file software.xlsx in the supplementary materials).

The transfer of local or remote data to the CINECA storage facility can be done either using: (i) Rsync or GridFTP exploiting the CINECA Intel QDR (40Gb/s) Infiniband high-performance network, for those sources with sufficient bandwidth, or (ii) sending by carrier portable storage devices to the CINECA user support team for those users with insufficient bandwidth. A schematic of this process is represented in Fig. 1.

\section{CINECA supercomputers facility}

CINECA continuously invests in state-of-the-art resources for computing and storage, usually opting for general purpose hardware compatible with the widest possible range of scientific domains. For this reason, the hardware setup (Table 1) of the computational environment available to ELIXIR-IT HPC@CINECA users has slightly changed over the years, mostly in order to provide the best possible available solutions. At the time of writing, HPC@CINECA users have access to two supercomputers, Marconi (Tier 0) and Galileo (Tier 1), available for production runs. Which one is assigned to each user depends on the resources required by the project. A third legacy cluster called Pico (Tier 1), which was the system initially reserved for the HPC@CINECA program back in 2016, is now reserved only to projects with particular high demands in terms of random access memory.

A more thorough description of these computational platforms is available from the CINECA portal. ${ }^{4}$ The CINECA data storage facility consists of arrays of high throughput devices (based on the GSS technology) for a total amount of about 4 PB of storage, connected to a large capacity tape library for a total actual amount of 12 PByte (expandable to 16 PByte).

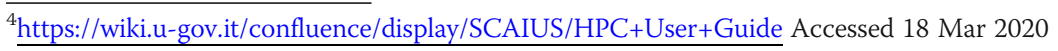


Table 1 Cineca high-performance computing clusters available for bioinformatic projects during the ELIXIR-IT HPC@CINECA call period, some providing higher-memory nodes. Projects are assigned to one cluster or the other upon technical evaluation from Cineca's staff and depending on the nature and needs of the project itself. Detailed instructions on how to get access to and use the clusters are provided to the PIs after projects approval and are also available on the Cineca website. Marconi A2 is going to be replaced by the Marconi 100 cluster in a few months

\begin{tabular}{lllll}
\hline HPC cluster & Nodes & Total core & RAM/Node (GB) & Architecture \\
\hline Pico (2015-2017) & 70 & 1400 & 128 & Intel Sandy-Bridge \\
Galileo & 516 & 16,512 & 128 & Intel Haswell + GPU \\
Marconi A2 & 3176 & 215,968 & 96 (cache mode) & Intel Knights Landing \\
\hline
\end{tabular}

All CINECA supercomputers use a common file system layout: shared areas complement the usual local HOME, SCRATCH and WORK private storage areas intended respectively for executables, big outputs and final outputs shared with the rest of the project team. A schematic and more detailed description of the file system of each cluster is reported in Fig. 2.

\section{Command line software environment}

A typical bioinformatics analysis pipeline requires the execution of complex workflows that can be logically represented as modules organized in a graph. These workflows often integrate several custom scripts as well as open source bioinformatics tools. Since software packages installation and maintenance can require considerable efforts by the user, the HPC@CINECA support team provides bioinformatics software within the CL environment through the "Environment Modules" UNIX package [10]. This solution allows users to easily load and unload tools, reference genomes and annotations required for their analyses within their own working environment. Table S1 reports the complete portfolio of currently available bioinformatics software for HPC@CINECA

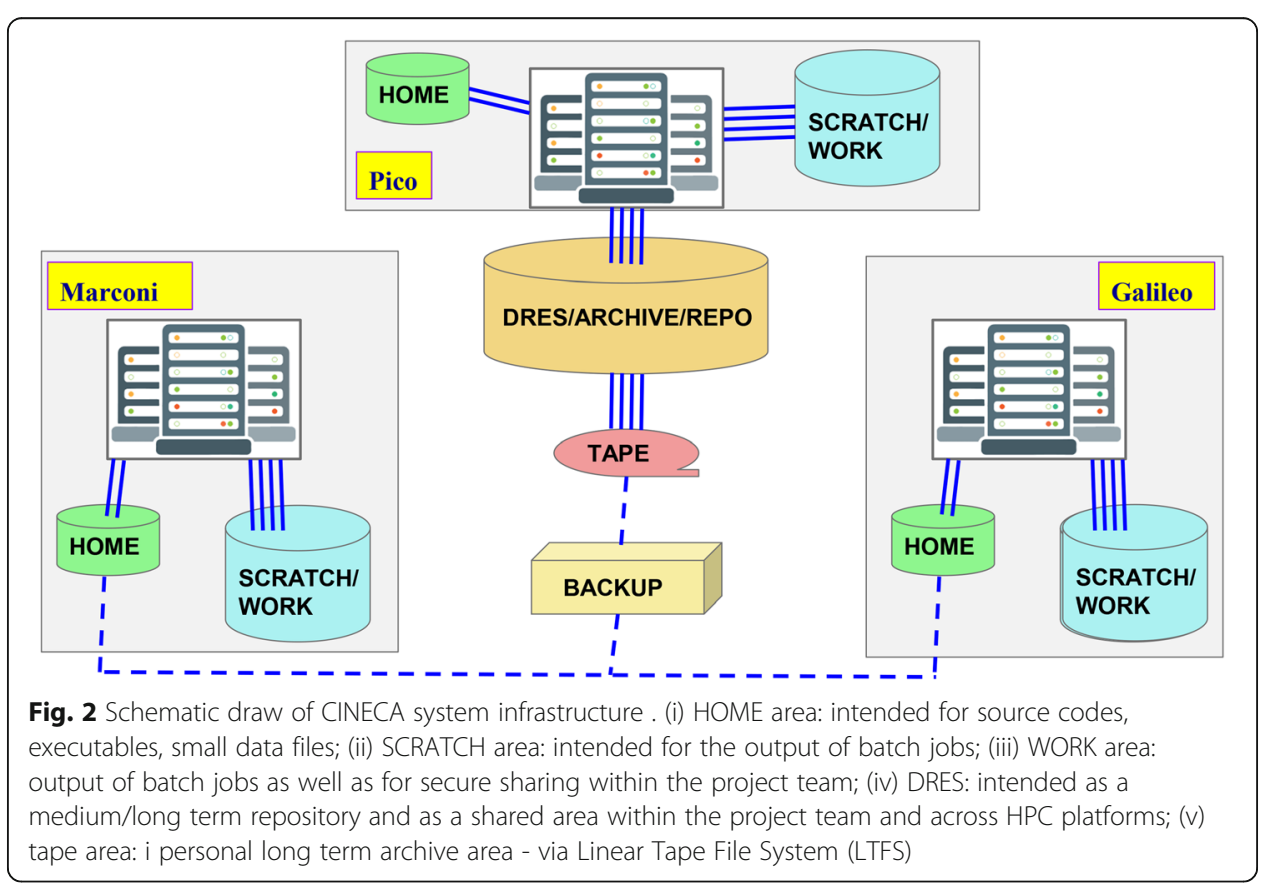


users. Users can always require the installation of additional software system-wide or add, on their own, software and custom scripts to their account.

Furthermore, each cluster currently incorporates reference datasets consisting of reference genome assemblies and annotation files for model organisms such as human (hg18, hg19, hg38); mouse (mm9, mm10); cow (bostau7, bostau8); horse (EquCab3); pig (Sus scrofa 11.1); rat (rn6) an so on, as well as a collection of meta-data for major commercial exome kits (Illumina, Agilent and Nimblegen).

At the time being the software environment is not GDPR compliant and therefore project applications involving the analysis of sensitive data have to be turned down.

\section{Scientific workflows}

ELIXIR-IT HPC@CINECA provides access also to fully automated, expert designed and highly optimized bioinformatics pipelines which allow to perform routine NGS data analysis through user-friendly web interfaces. Three different analysis workflows are currently available:

1) $\mathrm{CoVaCS}$ [11], a fully automated system for genotyping and variant annotation of resequencing data produced by second generation NGS technologies. CoVaCS offers state of the art tools for variant calling and annotation along with an expert made pipeline for the analysis of whole genome shotgun (WGS), whole exome sequencing (WES) and targeted resequencing data (TGS), performing all steps from quality trimming of the sequencing data to variant annotation and visualization. The final set of variants is obtained by forming a consensus call-set (2 out of 3 rule) from three different algorithms based on complementary approaches: Varscan, GATK and Freebayes. The system is currently available at this URL. ${ }^{5}$

2) RAP [12] a web interface based package that allows the execution of several operations on RNA-Seq data, including: quality control, alignment, abundance estimation and differential expression analysis at gene and transcript levels, differential alternative splicing and polyAdenilation, detection of fusion transcripts. The web interface of RAP is available at this URL. ${ }^{6}$

3) Expedit [13] is a web-service application dedicated to the exploration of RNA editing from human RNA-Seq data at preset or user-supplied specific genomic coordinates. Input data can be provided both in the form of raw reads (FASTQ or SRA format files) files, or in the form of aligned reads (in SAM/BAM format). The comparative analysis is carried on against a large collection of known editing sites collected in the DARNED database [14] as well as other user-provided potentially edited positions. Final results are displayed as custom tracks at the University of

5https://bioinformatics.cineca.it/covacs Accessed 18 Mar 2020

6 https://bioinformatics.cineca.it/rap/ Accessed 18 Mar 2020 
California, Santa Cruz (UCSC) genome browser, for a quick examination of the genomic context. ExpEdit is freely available at the following link. ${ }^{7}$

\section{Evaluation of the project proposals and user support}

Project proposals submitted to the ELIXIR-IT HPC@CINECA program are initially evaluated by a scientific and technical board, appointed by ELIXIR-IT, to ensure the scientific soundness and technical feasibility of the application.

Projects are evaluated with a "first come, first served" policy until the total annual resource budget of the program, consisting of over $1 \mathrm{M}$ core hours, has been assigned. Applications are evaluated within seven working days from submission, while approved applications obtain access to the computational resources within further seven working days from acceptance.

Users associated to a project are classified either as principal investigator (PI), typically who submitted the project proposal, or as part of a PI-managed set of authorized users. The standard package allocated to each approved project consists of $50 \mathrm{k}$ core hours and $1 \mathrm{~TB}$ of permanent storage, for a duration of 6 months, extensions of the projects deadline and requests of additional resources are considered on a case-by-case basis taking into account their scientific merit.

The jobs submission procedure makes use of the free and open-source job scheduler SLURM, a computer application for controlling unattended background program execution of jobs. Jobs requiring more than $10 \mathrm{~min}$ of core/hours execution need to be executed exclusively using SLURM directives. The job scheduler of the CINECA's HPC multi-user environment has been configured so that each user is allowed no more than 20 jobs at the same time. The core hours employed by users associated to a project are, in turn, subtracted from the core hours budget of the project itself.

To help users dealing with the complexity of the command line interface and of the job scheduler, CINECA makes available a user support team always readily available to help researchers in:

- Setting up and enriching the software environment, with the quick deployment of any additional module required by a project;

- Automating the analysis of large amounts of data, with fine-tuned, cluster-specific, configuration of parameters for the different platforms;

- Helping in optimizing the analysis of big-data experiments;

- Collaborating in the development of highly parallelized code (through MPI, python, $R$, etc.) suitable for HPC infrastructures;

- Automating the bioinformatics pipelines via SLURM directives.

\section{Results}

Since its launch on april 2016, ELIXIR-IT HPC@CINECA has provided access to HPC resources to 63 research projects, allocating a total of approximately 3,250,000 CPU core hours. The 63 projects are distributed among 28 research centres (Universities and Research Centers). Figure 3a reports the distribution of proposed projects over several biological macro-areas, showing as the initiative engaged researchers from different 
backgrounds and different needs in terms of computational requirements and software. As shown in Fig. 3b, the number of projects submitted is growing constantly since the first opening of the call. The growth can be ascribed both to dissemination activities performed in several national conferences $[15,16]$ as well as to the good feedback obtained from the first participants, as demonstrated by the publication of the results of HPC@CINECA research projects in peer reviewed scientific journals.

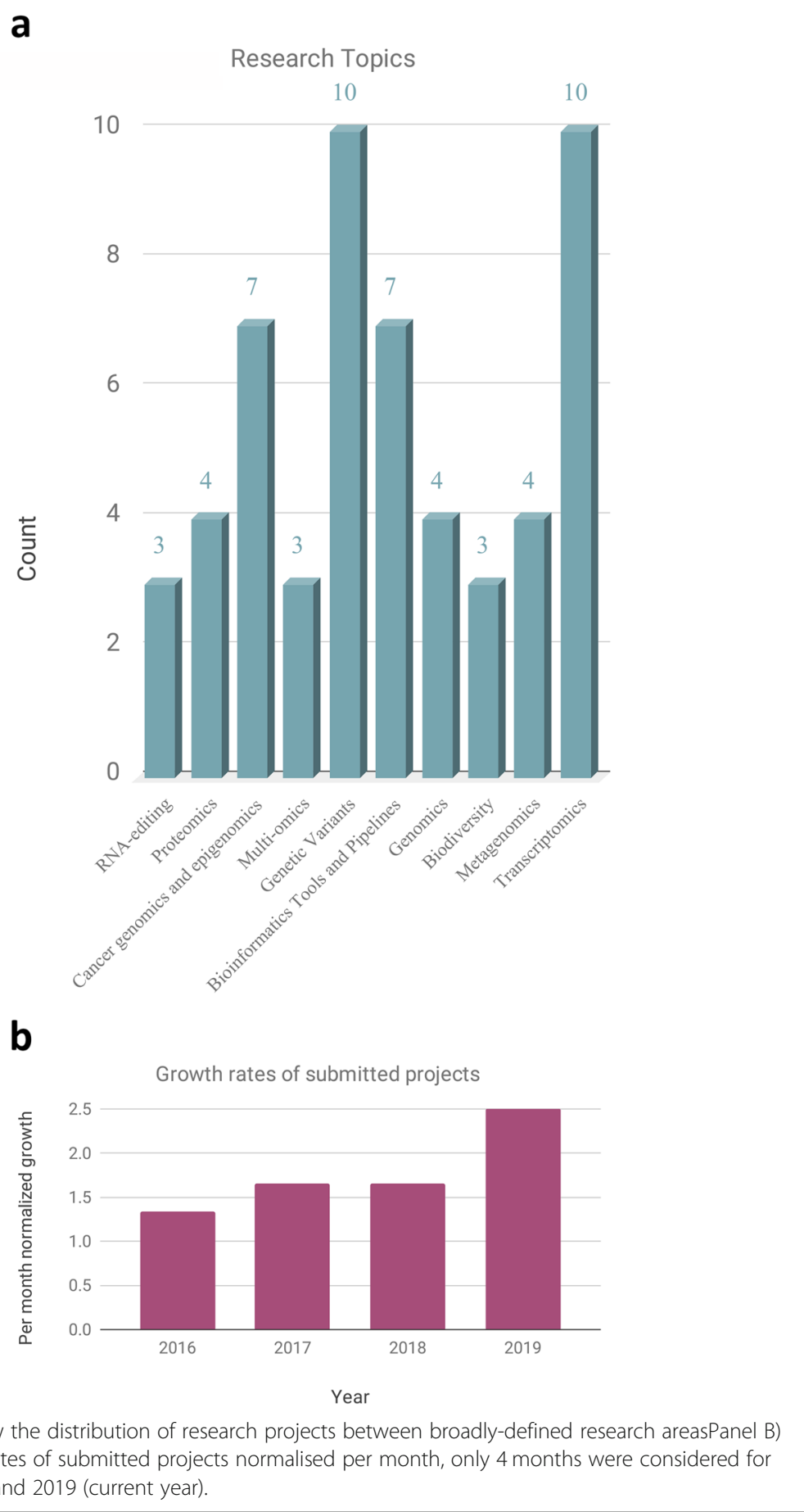




\section{Use cases}

Here we provide a brief summary of some research projects that were successfully completed thanks to the HPC resources provided by the ELIXIR-IT HPC@CINECA call.

\section{Genome-wide mapping of 8-oxo-7,8-dihydro-2'-deoxyguanosine across human genome}

8-Oxo-7,8-dihydro-2'-deoxyguanosine (8-oxodG) is one of the major DNA modifications that occurs when the DNA is exposed to pro-oxidant species (ROS) generated by endogenous metabolism. 8-oxodG is a potent premutagenic lesion for its ability to pair with both cytosine and adenine residues, thus causing $\mathrm{G}: \mathrm{C}$ to $\mathrm{T}: \mathrm{A}$ transversions during DNA replication $[17,18]$.

Several thousand residues of 8-oxodG are constitutively produced in the genome of mammalian cells and a new method has been developed to identify their genomic distribution.

Recently, by using OxiDIP-Seq, Amente et al. [amenteetaloxidip19] reported the genome-wide distribution of 8-oxodG in proliferating DDR-proficient mammary cells (MCF10A and MEFs). Analysis of OxiDIP-Seq revealed that endogenous 8-oxodG is regioselective distributed across the mammalian genome. Moreover, an integrated data analysis starting from OxiDIP-Seq, ChIP-Seq anti-gH2AX, ChIP-Seq anti-POLII, GRO-Seq and RNA-Seq led to the identification of an accumulation of endogenous DNA damage within the gene body of long genes with poor-to-moderate transcription levels. In terms of computational resources we used $2 \mathrm{~TB}$ of permanent storage and $200 \mathrm{k}$ core/hours to perform all the analysis of about $500 \mathrm{~GB}$ of starting input data. They were analyzed using HPC@CINECA computer resources,) through both the command line environment and the bioinformatics automated pipelines [refs. To RAP and CAST] developed and provided by the CINECA-ELIXIR IT team. This computational effort led to further insights about the molecular mechanisms underlying the heterogeneity of the local mutation rate and the understanding of why certain regions seem to be more, while others less, prone to oxidation. A full description of this work can be found in [19].

\section{New HPC-optimized algorithm for prediction of RNA-editing events from RNA-Seq data}

RNA editing is a relevant epitranscriptome modification occurring in a wide range of organisms. In humans, it affects nuclear and cytoplasmic transcripts mainly by the deamination of adenosine (A) to inosine (I) through ADAR enzymes acting on double RNA strands [LiChurch13]. RNA editing has a plethora of biological effects and its deregulation has been linked to a variety of human diseases including psychiatric, neurological and neurodegenerative disorders, and cancer [20]. Several bioinformatics tools to investigate RNA editing events in NGS data have been released [21]. However, its computational identification is a highly time-consuming process, requiring the traversing of very large alignments files in BAM format, position-by-position. Employing ELIXIR-IT HPC@CINECA resources the original REDItools package [22], one of the most accurate tools to call RNA editing events in RNA-Seq experiments [21], the A-to-I calling process has been speeded up, optimizing its implementation for HPC infrastructures:

- a first optimization in the new version of the code, REDItools2.0, consisted in loading the sequences from disk by reading each sequence only once, keeping it in 
memory until no longer than needed. This implementation was on average 8-10 times faster than the original version running on a single core;

- another improvement of the algorithm consisted in optimizing the splitting of the genome into genomic intervals. The initial release of REDItools treated equally different chromosomal regions, by dividing the whole genome in chunks of equal size and assigning each chunk to a thread. Since usually expression data do not exhibit a constant coverage, the number of reads per genomic unit (density of mapped reads) is quite variable and the original version of REDItools spent a lot of computational time in high-density genomic regions. We therefore implemented an optimal interval division in order to guarantee an approximately uniform per-thread workload;

- a parallel version of REDItools 2.0 has also been implemented by writing an ad-hoc MPI Python script based on the use of the mpi4py library [23]. This library provides binding of the Message Passing Interface (MPI) standard for the Python programming language. In this way it is possible to exploit multiple computing nodes by means of collective communication MPI primitives. A simple master/slave template has finally been implemented for coordinating the overall computation.

Executions of the optimized algorithm on real RNA-Seq experiments have shown that the novel REDItools2.0, is on average ten times faster than the previous implementation and the speed up scales adequately with the number of cores involved in the analysis (Fig. 4) thus representing the first HPC resource specifically devoted to RNA-editing detection.

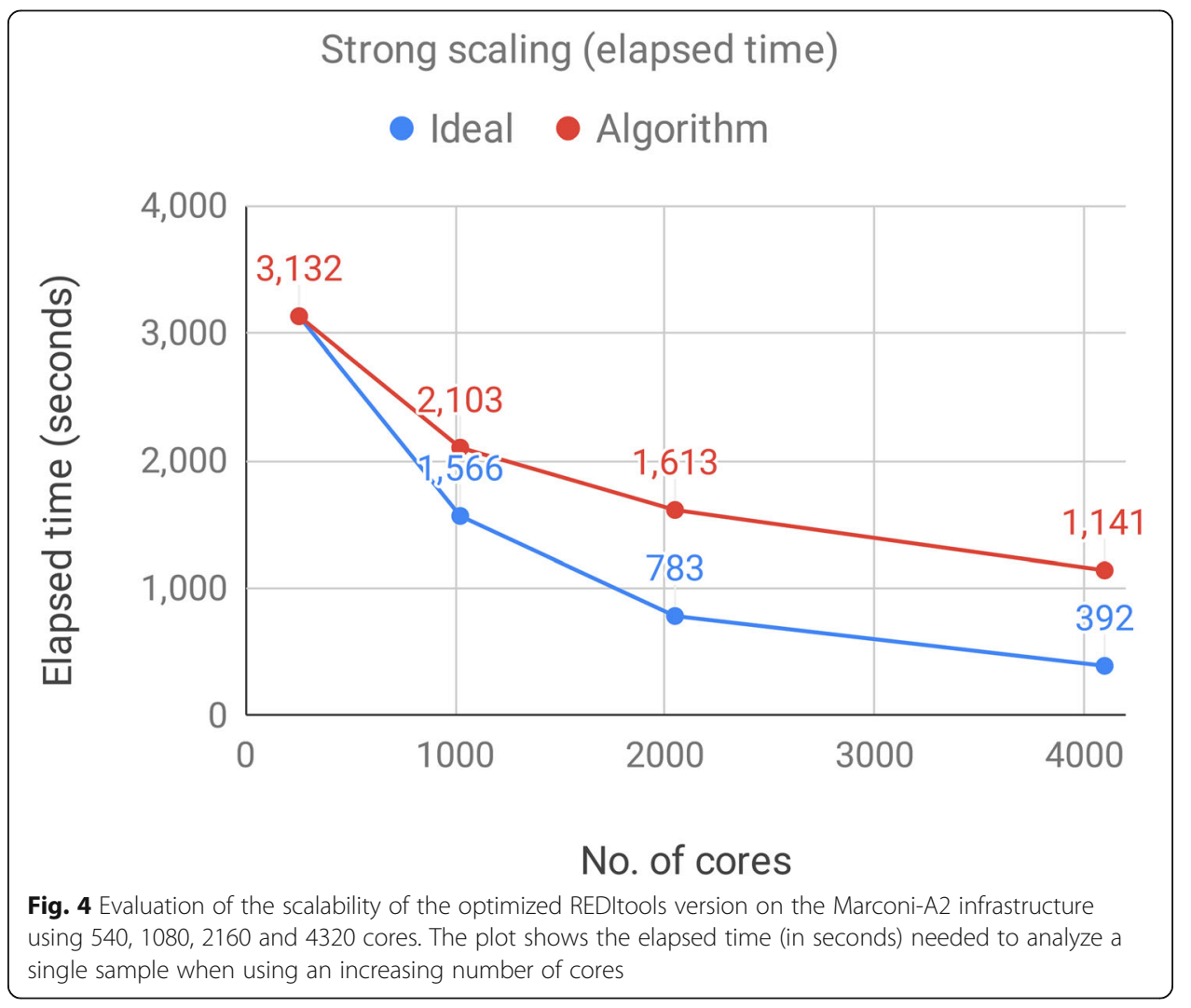


Thanks to the algorithmic optimization described above, the novel REDItools2.0 package has been then used to investigate RNA editing in very large cohorts of RNAseq experiments like those produced in GTEx or TCGA projects after the award of additional resources through a competitive PRACE (Partnership for Advanced Computing in Europe) project (ProjectID: 2016163924 GREaT - Genome wide identification of RNA editing sites in very large cohorts of human whole transcriptome data). Full description of this work is available in PRACE White Paper. ${ }^{8}$

\section{Creation of a comprehensive database for genomics data in peach (P. persica L. Batsch)}

Peach is an economically important fruit tree species of temperate region. Integrating novel genomics tools is a fundamental goal for increasing the efficiency of breeding activities and the leveraging of basic knowledge in this species. After the release of the first peach genome draft, the remarkable advances in high-throughput molecular tools has led to the generation of a multitude of genomics data from several whole-genome re-sequencing projects.

In this project, Whole-genome sequencing data of 125 peach ( $P$. persica L. Batsch) accessions and 21 wild relatives of the Amygdalus subgenus have been downloaded from the NCBI SRA [24] for a whole of 146 accessions publicly available (input data size about $10 \mathrm{~TB})$. Variant discovery was achieved by applying an imputation-free joint variant-calling procedure on the 146 accessions, improving variant discovery by leveraging population-wide information from a cohort of multiple samples [25]. $200 \mathrm{k}$ core/ hours have been used to analyse all the samples on the Pico cluster to create the compendium dataset of peach variants. The identified peach variants, both SNP and InDels, are available at the PeachVar-DB portal ${ }^{9}$ that provides an easy access to the information mined from peach Whole Genome Re-Sequencing (WGRS) data. Full description of this work can be found in [26].

\section{High-quality genome assembly for the European barn swallow (Hirundo rustica rustica)}

The barn swallow is a passerine bird with at least eight recognized subspecies in Europe, Asia, and North America. Due to its synanthropic habits and its cultural value, the barn swallow is also a flagship species in conservation biology [27]. The availability of high-quality genomic resources, including a reference genome, is thus pivotal to further boost the study and conservation of this species. To facilitate further population genetics and genomic studies, as a part of the Genome10K effort on generating high-quality vertebrate genomes (Vertebrate Genomes Project) [28].

Formenti et al. [29] have assembled a highly contiguous genome assembly using single molecule real-time (SMRT) DNA sequencing and Bionano optical map technologies for the European subspecies (Hirundo rustica rustica). The assembly of the genome, which was performed entirely on the Marconi CINECA HPC supercomputer occupied 3840 central processing unit (CPU) hours and a total amount of $2.2 \mathrm{~Tb}$ of random access memory (RAM) for reads correction, $768 \mathrm{CPU}$ hours and $1.1 \mathrm{~Tb}$ of RAM for the trimming steps, and $3280 \mathrm{CPU}$ hours and $2.2 \mathrm{~Tb}$ of RAM for the assembly phase. The entire process was completed in less than 5 days on the CINECA HPC platform, while

${ }_{8}^{8}$ https://prace-ri.eu/wp-content/uploads/WP258.pdf Accessed 18 Mar 2020

9 http://hpc-bioinformatics.cineca.it/peach/main Accessed 18 Mar 2020 
re-analysis of the same data on a local server required more than 80 days (Matteo Chiara, personal communication) at full computational capacity.

After removal of haplotigs, the final assembly resulted in approximately $1.21 \mathrm{Gbp}$ in size, with a scaffold N50 value of more than $25.95 \mathrm{Mbp}$, representing a considerable improvement over the previously reported assembly [30]. Systematic comparisons of this high quality draft genome assembly of $H$. rustica with a collection of closely and distantly related bird genomes provide phylogenomics profiles of structural rearrangements and gene losses/gene duplications. The approach used for the assembly of the barn swallow genome, while attesting to the effectiveness of SMRT sequencing combined with DLS optical mapping for the assembly of vertebrate genomes, provides an invaluable asset for population genetics and genomics in the barn swallow and for comparative genomics in birds. Full description of this work can be read in [29].

\section{Massive NGS data analysis reveals hundreds of potential novel gene fusions in human cell lines}

One of the genetic alterations that are linked to cancer development in addition to single nucleotide mutations are gene fusions deriving from chromosome rearrangements. The availability of sequence data from NGS techniques has made possible the discovery of a huge amount of such alterations. However, current algorithms for fusion detection either have high false positive result rates or miss some real events. Hence, it is very important to be able to run and compare the results of several algorithms, with different discovery properties.

Gioiosa et al. [31] have extensively carried out the analysis of 935 paired-end RNAsequencing experiments downloaded from the Cancer Cell Line Encyclopedia repository (CCLE) ${ }^{10}$ for a total of $32 \mathrm{~TB}$ of input raw data. The aim was addressing novel putative cell-line specific gene fusion events in human malignancies. Four gene fusion detection algorithms were launched on the CCLE samples to detect gene fusion events, for a total of $500 \mathrm{k}$ core/hours. Furthermore, a prioritization analysis was performed by running a Bayesian classifier that adds an in silico validation on detected events. The collection of fusion events supported by all of the predictive algorithms provides a robust dataset of $\sim 1700$ in silico novel candidates among gene fusion events. These data have been stored, collected and integrated with other external resources within the LiGeA portal (cancer cell LInes Gene fusion portAl), ${ }^{11}$ where they are browsable and freely downloadable. Full description of this work can be found in [31].

\section{Discussion}

\section{HPC perspectives for the bioinformatics community}

CINECA is strongly focused on understanding and collecting all the needs of researchers in the bioinformatics field. Besides the HPC systems involved in the ELIXIRIT HPC@CINECA pilot call, multiple prototype systems are provided to be tested with new test cases. For example, systems with hierarchical memory equipped with fast high bandwidth memory (HBM), standard DDR4 RAM and newly available non-volatile memory (NVM). The latter is especially interesting for bioinformatics test cases since it

\footnotetext{
${ }^{10}$ https://portals.broadinstitute.org/ccle Accessed 18 Mar 2020

${ }^{11}$ http://hpc-bioinformatics.cineca.it/fusion/ Accessed 18 Mar 2020
} 
can provide cost effective solutions with TBs of RAM memory [MC1] with performance comparable with standard DDR4. Challenging bioinformatics problems that can benefit from the use of High-Performance computational resources requiring tremendous RAM availability regards:

- metagenomics research in which scientists need to analyze over a million metagenomes in search of signature genes that could serve important functions in biomanufacturing for health, energy, and industry.

- large genome assembling with PacBio single molecule sequencing reads.

- analysis of large cohorts of individuals (> 10,000 samples) with Genome Wide Association Study (GWAS) techniques.

CINECA will also devote considerable efforts in keeping the HPC infrastructure up to date with the most advanced technological solutions that will become available in the forthcoming years. In this regard, Fig. 5 shows the roadmap of future development of CINECA HPC systems in the timeframe 2020-2027: different routes may be taken but the clear goal is to provide researchers with a world class exascale HPC systems by 2025-2027. In particular, pre-exascale systems may be available as early as 2020 through the EuroHPC European initiative [32] or alternatively, in the following years, through Italian national funding. A typical computationally demanding application, which could potentially benefit from exascale architecture, could be represented by personalized medicine, since in this field the celerity of data analysis can be crucial for saving people's lives.

Indeed, computational challenges for personalized medicine usually pursue the objectives of:

- providing systems for handling and analysing heterogeneous patients' data and clinical questions, for example through the implementation of new parallel deep learning algorithms;

- developing optimized, secure and law-compliant personalized medicine computational pipelines;

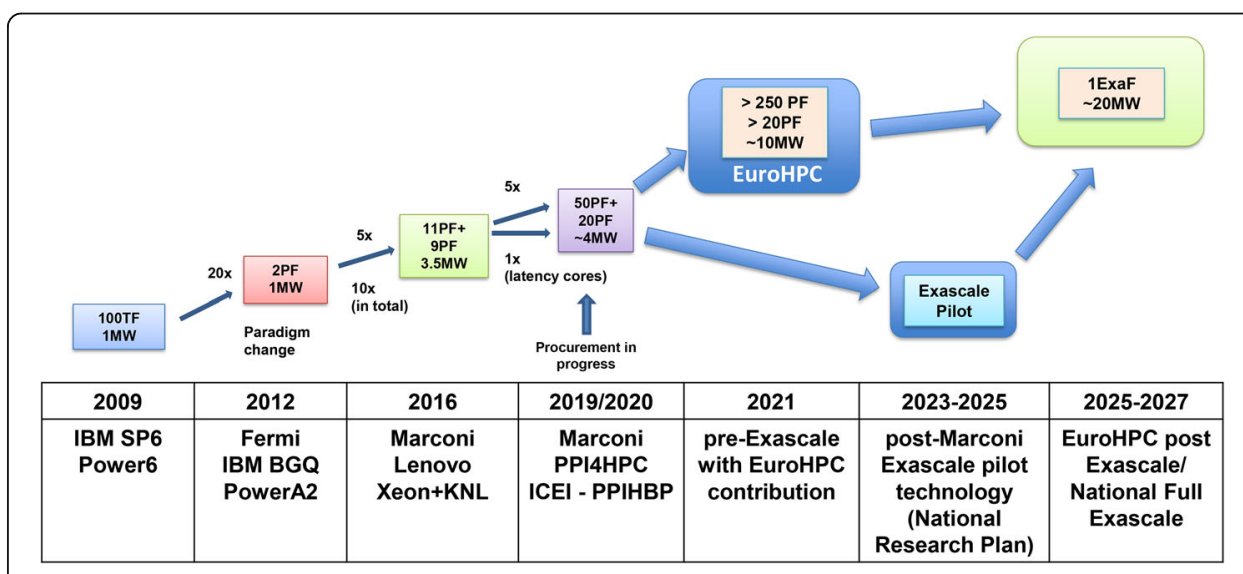

Fig. 5 CINECA roadmap towards systems of exaflop capabilities 
- modelling and predicting drug response, understanding the molecular basis of key cellular interactors and speeding up the extraction of information from millions of disease patient records to determine optimal disease treatment strategies;

- producing and testing all the components necessary to exploit Exascale calculation to generate patient models.

Moreover, a convergence between traditional HPC and artificial intelligence (AI) is planned in the first Exascale platform to be installed in the USA at the Argonne National Laboratory in 2021. Bioinformatic is going to fully take advantage of this convergence, with new applications not only in personalized medicine but in the full range of molecular biology research.

From the user standpoint, the increase in computational power has to match the usability of the systems. To this extent, it is clear the importance of the work of CINECA staff described in this paper. We foresee in the future a more data-centric approach in using the HPC resources, where data stored in the system are subject to multiple analysis, possibly involving a range of technological solutions, such as computing accelerators (NVIDIA, AMD GPUs), specific services hosted in virtual machines (OpenStack, Cloud solutions), fast and big-mem memory nodes (HBM, NVM), and many others.

\section{Conclusions}

In this paper, we describe a pilot program for provisioning HPC resources to bioinformatics researchers with the aim of providing a viable solution for large scale analyses of massive NGS data, a need that is becoming increasingly common in the biomedical research community.

At the beginning of massive sequencing advent, the analysis software used only a single core and no parallel programming techniques, such as threading or MPI. This has changed over the years and many current applications take advantage of the multithreading modality in order to accelerate the calculations.

In summary, having a good mix of general purpose computational power, the possibility of booking an arbitrary number of cores (multi-threading) or nodes for the jobs that require more CPUs, or very large memory nodes for jobs with large memory requirement, has been shown to be a very efficient and affordable infrastructure for bioinformatic data analysis. As a confirmation of this trend, four out of the five success stories reported in the "Result" paragraph ( $a, c, d, e$ test cases) were optimized on cluster machines by using a multi-threading approach for most of the programs used by the applied pipelines, and MPI optimization was used in the remaining test case (b).

Currently the ELIXIR-IT HPC@CINECA program constitutes, at the best of our knowledge, a unique initiative in the bioinformatics landscape since it is open to smallto-medium sized projects from all European life science researchers based only on the technical and scientific soundness of their applications. Usually, the access model to HPC resources for bioinformatics is bound to serve researchers from specific institutions or collaborating to specific projects, or require researchers to win competitive calls, like PRACE, usually reserved to projects of much greater scale (millions of core hours per project). Actually, the utility of the program has been not only to allow the execution of complex bioinformatic analyses, but also to provide its maintainers with insights on how to organise and manage one of the few public High Performance 
Computing platforms dedicated specifically to bioinformatics in Europe, and the only one of this type available in Italy. Since the needs of the bioinformatics community in terms of computational and storage resources in the coming few years will rise steadily, this initiative can represent a valid model for the provisioning of HPC services also by other ELIXIR Nodes or similar infrastructural entities in the near future. As one of the key technological partners of ELIXIR-IT, CINECA continuously explores possible actions to improve the technological infrastructure and provide valuable computational services to European life scientists and bioinformaticians. On their part, ELIXIR-IT and CINECA will continue their commitment to this initiative, possibly expanding it with new features and integrating the HPC platform with other services.

\section{Availability and requirements}

Project name: ELIXIR-IT HPC@CINECA.

Project home page: http://www.beaconlab.it/HPC-cineca

Operating system(s): Platform independent.

Programming language: Not applicable.

Other requirements: SSH client.

License: Not applicable.

Use of the platform is subject to approval (see http://www.beaconlab.it/HPC-cineca).

\section{Supplementary information}

Supplementary information accompanies this paper at https://doi.org/10.1186/s12859-020-03565-8.

Additional file 1 Table S1: list of the bioinformatics software available for HPC@CINECA users on the three computational platforms (PICO, MARCONI and GALILEO) updated to May 2019. The software portfolio reflects the needs of the users since 2016 and is gradually enriched to meet the requirements of newly approved projects.

\section{Abbreviations}

NGS: Next Generation Sequencing; HPC: High Performance Computing; ELIXIR-IT: The Italian Node of ELIXIR; HTS: High throughput sequencing; JRU: Joint Research Unit; WGS: Whole genome shotgun; WES: Whole exome sequencing; TGS: Targeted genome re-sequencing; PI: Principal investigator; 8-oxodG: 8-Oxo-7,8-dihydro-2'-deoxyguanosine

\section{Acknowledgements}

We would like to thank all HPC@Cineca users for their wonderful feedback. GC acknowledges the support by the "Departments of Excellence-2018" Program (Dipartimenti di Eccellenza) of the Italian Ministry of Education, University and Research, DIBAF-Department of University of Tuscia, Project "Landscape 4.0 - food, wellbeing and environment".

\section{About this supplement}

This article has been published as part of BMC Bioinformatics Volume 21 Supplement 10, 2020: Proceedings from the 13th Bioinformatics and Computational Biology International Conference - BBCC2018. The full contents of the supplement are available online at https://bmcbioinformatics.biomedcentral.com/articles/supplements/volume-21-supplement-10 .

\section{Authors' contributions}

GP, TC, GC, and FZ conceived the ELIXIR-IT HPC@Cineca service. TC, TF, GC and SG developed the command line environment. MC and MAT provided technological support from the CINECA and ELIXIR-IT side respectively. SG, EP, MC, MF SA, and MC led the HPC@CINECA projects reported in the use cases section and wrote the corresponding paragraphs. MC, TC, GC and FZ wrote the rest of the manuscript. TC, GC, and FZ coordinated the service and supervised all related activities. All authors read the manuscript and suggested improvements. The authors read and approved the final manuscript.

\section{Funding}

This work has been supported by ELIXIR-IIB and CINECA internal funding and the H2020 ELIXIR-Excelerate (RIA 676559), EOSC-Life (RIA 824087) and EOSC-Pillar (RIA 857650) projects. Publication costs are funded by ELIXIR-Excelerate. The funder played no role in the design of the study and collection, analysis, and interpretation of data and in writing the manuscript. 
Ethics approval and consent to participate

Not applicable.

\section{Consent for publication}

Not applicable.

\section{Competing interests}

The authors declare that they have no competing interests.

\section{Author details}

${ }^{1}$ Department of Ecological and Biological Sciences (DEB), University of Tuscia, Viterbo, Italy. ${ }^{2}$ CINECA, SuperComputing Applications and Innovation Department, Rome, Italy. Institute of Biomembranes, Bioenergetics and Molecular Biotechnologies, National Research Council (IBIOM-CNR), Bari, Italy. ${ }^{4}$ Department of Biosciences, Biotechnology and Biopharmaceutics, University of Bari "A. Moro", Bari, Italy. ${ }^{5}$ Department of Biosciences, University of Milan, Milan, Italy. ${ }^{6}$ IRCCS-Istituto di Ricerche Farmacologiche "Mario Negri", Milano, Milan, Italy. ${ }^{7}$ Department of Molecular Medicine and Medical Biotechnologies, University of Naples 'Federico II', Naples, Italy. ${ }^{8}$ Department of Agricultural and Environmental Sciences - Production, Landscape, Agroenergy (DISAA), University of Milan, Milan, Italy. ${ }^{9}$ Department for Innovation in Biological, Agro-food and Forest systems (DIBAF), University of Tuscia, Viterbo, Italy.

\section{Published: 25 August 2020}

\section{References}

1. Goodwin S, McPherson J, McCombie W. Coming of age: ten years of next-generation sequencing technologies. Nat Rev Genet. 2016;17:333-51.

2. Wang Z, Gerstein M, Snyder M. RNA-Seq: a revolutionary tool for transcriptomics. Nat Rev Genet. 2009;10:57-63

3. Rubelt F, Busse C, Bukhari $S$, et al. Adaptive immune receptor repertoire community recommendations for sharing immune-repertoire sequencing data. Nat Immunol. 2017;18:1274-8.

4. Tang F, Barbacioru C, Wang Y, et al. mRNA-Seq whole-transcriptome analysis of a single cell. Nat Methods. 2009;6:377-82.

5. Nakato R, Shirahige K. Recent advances in ChIP-seq analysis: from quality management to whole-genome annotation. Brief Bioinform. 2017;18(2):279-90.

6. Marx V. Biology: the big challenges of big data. Nature. 2013;498(7453):255-60.

7. Stephens ZD, Lee SY, Faghri F, Campbell RH, Zhai C, Efron MJ, et al. Big data: astronomical or Genomical? PLoS Biol. 2015;13:e1002195.

8. Lampa S, Dahlö M, Olason PI, Hagberg J, Spjuth O. Lessons learned from implementing a national infrastructure in Sweden for storage and analysis of next-generation sequencing data. Gigascience. 2013;2(1):9. https://doi.org/10.1186/ 2047-217X-2-9.

9. Rajasekar A, Russell T, Coposky J, de Torcy A, Xu H, Wan M, et al. The integrated rule-oriented data system (iRODS 4.0) microservice workbook; 2015. p. 248.

10. Furlani JL. Modules: providing a flexible user environment. San Diego: Proceedings of the Fifth Large Installation Systems Administration Conference (LISA V); 1991. p. 141-52. September 30-October 3.

11. Chiara M, Gioiosa S, Chillemi G, D'Antonio M, Flati T, Picardi E, et al. CoVaCS: a consensus variant calling system. BMC Genomics. 2018;19(1):120. https://doi.org/10.1186/s12864-018-4508-1.

12. D'Antonio M, D'Onorio De Meo P, Pallocca M, Picardi E, D'Erchia AM, Calogero RA, et al. RAP: RNA-Seq analysis pipeline, a new cloud-based NGS web application. BMC Genomics. 16(Suppl 6):S3. https://doi.org/10.1186/1471-2164-16-S6-S3 Epub 2015 Jun 1. PubMed PMID: 26046471; PubMed Central PMCID: PMC4461013.

13. Picardi E, D'Antonio M, Carrabino D, Castrignanò T, Pesole G. ExpEdit: a webserver to explore human RNA editing in RNA-Seq experiments. Bioinformatics. 2011;27(9):1311-2.

14. Kiran A, Baranov PV. DARNED: a DAtabase of RNa EDiting in humans. Bioinformatics. 2010;26:1772-6.

15. Presenter GS. HPC bioinformatics resources for ELIXIR scientific community. Cagliari: Presented at the meeting of BITS Bioinformatics Italian Society; 2017.

16. Presenter $\subset \mathrm{C}$. Managing big data bioinformatics on shared high-performance computing platforms. Naples: Presented at the meeting of BBCC2018 bioinformatics and computational biology conference; 2018, November.

17. Cooke MS, Evans MD, Dizdaroglu M, Lunec J. Oxidative DNA damage: mechanisms, mutation, and disease. FASEB J. 2003;17:1195-214.

18. Agnez-Lima LF, Melo JTA, Silva AE, Oliveira AHS, Timoteo ARS, Lima-Bessa KM, et al. DNA damage by singlet oxygen and cellular protective mechanisms. Mutat Res - Rev Mutat Res. 2012;751:15-28.

19. Amente S, Di Palo G, Scala G, Castrignanò T, Gorini F, Cocozza S, et al. Genome-wide mapping of 8-oxo-7,8-dihydro-2'deoxyguanosine reveals accumulation of oxidatively-generated damage at DNA replication origins within transcribed long genes of mammalian cells. Nucleic Acids Res. 2019;47(1):221-36. https://doi.org/10.1093/nar/gky1152 Epub 2018 Nov 20. PubMed PMID: 30462294; PubMed Central PMCID: PMC6326803.

20. Maas S, Kawahara Y, Tamburro KM, Nishikura K. A-to-I RNA editing and human disease. RNA Biol. 2006;3(1):1 -9. https:// doi.org/10.4161/rna.3.1.2495 Epub 2006 Jan 12. PubMed PMID: 17114938; PubMed Central PMCID: PMC2947206.

21. Diroma MA, Ciaccia L, Pesole G, Picardi E. Elucidating the editome: bioinformatics approaches for RNA editing detection. Brief Bioinform. 2019;20(2):436-47. https://doi.org/10.1093/bib/bbx129.

22. Picardi E, Pesole G. REDItools: high-throughput RNA editing detection made easy. Bioinformatics. 2013;29(14):1813-4. https://doi.org/10.1093/bioinformatics/btt287 Epub 2013 Jun 5.

23. Dalcin L, Kler P, Paz R, Cosimo A. Parallel distributed computing using python. Adv Water Resour. 2011;34(9):1124-39. https://doi.org/10.1016/j.advwatres.2011.04.013.

24. Leinonen R, Sugawara H, Shumway M, International Nucleotide Sequence Database Collaboration. The sequence read archive. Nucleic Acids Res. 2011;39:D19-21. 
25. Liu X, Han S, Wang Z, Gelernter J, Yang B-Z. Variant callers for next-generation sequencing data: a comparison study. PLoS One. 2013;8:e75619.

26. Cirilli M, Flati T, Gioiosa S, Tagliaferri I, Ciacciulli A, Gao Z, et al. PeachVar-DB: a curated collection of genetic variations for the interactive analysis of peach genome data. Plant Cell Physiol. 2018;59:1-9ISSN: 0032-0781. https://doi.org/10. 1093/pcp/pcx183.

27. Turner A. The barn swallow. London: T \& AD Poyser; 2006. https://www.bloomsbury.com/uk/the-barn-swallow9780713665581/

28. Hered J. Genome 10K: a proposal to obtain whole-genome sequence for 10,000 vertebrate species. 2009;100(6):659-74. https://doi.org/10.1093/jhered/esp086 Epub 2009 Nov 5.

29. Formenti G, Chiara M, Poveda L, Francoijs K, Bonisoli-Alquati A, Canova L, et al. SMRT long reads and direct label and stain optical maps allow the generation of a high-quality genome assembly for the European barn swallow (Hirundo rustica rustica). Gigasciece. 2018;8(1) 2019:giy142. https://doi.org/10.1093/gigascience/giy142.

30. Safran RJ, Scordato ES, Wilkins MR, et al. Genome-wide differentiation in closely related populations: the roles of selection and geographic isolation. Mol Ecol. 2016;25(16):3865-83. https://doi.org/10.1111/mec.13740. Accessed 9 May 2020.

31. Gioiosa S, Bolis M, Flati T, et al. Massive NGS data analysis reveals hundreds of potential novel gene fusions in human cell lines. Gigascience. 2018;7(10):giy062. https://doi.org/10.1093/gigascience/giy062. Accessed 9 May 2020.

32. European Commission - Press release "Council backs Commission's plans to invest $€ 1$ billion in world-class European supercomputers", 2018, http://europa.eu/rapid/press-release_IP-18-5864_en.htm Date Accessed: 18 Mar 2020.

\section{Publisher's Note}

Springer Nature remains neutral with regard to jurisdictional claims in published maps and institutional affiliations.

Ready to submit your research? Choose BMC and benefit from:

- fast, convenient online submission

- thorough peer review by experienced researchers in your field

- rapid publication on acceptance

- support for research data, including large and complex data types

- gold Open Access which fosters wider collaboration and increased citations

- maximum visibility for your research: over $100 \mathrm{M}$ website views per year

At $\mathrm{BMC}$, research is always in progress.

Learn more biomedcentral.com/submissions 